\title{
A Study on a Single-Phase HTS Transformer with a Cylindrical Central Iron Core
}

\author{
Meiying Yin ${ }^{1, a}$, Yong Zhao ${ }^{2}$, Zhuyong $\mathrm{Li}^{3}$, Xingmei $\mathrm{Li}^{4}$, Wenzhao Yin ${ }^{4}$, Lan Duan ${ }^{4}$, Zihong Wang ${ }^{4}$, \\ Feng Zhao and Bin $\mathrm{Han}^{4}$ \\ ${ }^{1}$ Sino-Korean School of Multimedia Design, Shanghai University of Engineering Science, Shanghai, China \\ ${ }^{2}$ General Department of Power Grid Engineering Branch, China Energy Engineering Group Anhui Electric Power \\ Design Institute Company Ltd., Hefei, China \\ ${ }^{3}$ Department of Electrical Engineering, Shanghai Jiao Tong University, Shanghai, China \\ ${ }^{4}$ Dali Power Supply Bureau repair Institute, Dali City, Yunnan, China
}

\begin{abstract}
During the past decade, a number of high temperature superconducting (HTS) transformer prototypes have been designed, and the majority of them are full-core transformers and air-core transformers. However, in this study, we proposed a new type of HTS transformer, where the iron core is different in that the limbs and connecting yokes are absent. To investigate the performance of this type of HTS transformer, we design and fabricate a single-phase HTS partial core transformer prototype using the secondary generation $(2 \mathrm{G})$ HTS GdBCO wires. The iron core is at room temperature, and the windings are immersed in liquid Nitrogen. The structures of primary and secondary windings are three layers connected in series and nine double pancakes connected in parallel, respectively. Fundamental characteristics are obtained by standard short-circuit, no-load and load tests in liquid Nitrogen temperature of $77 \mathrm{~K}$. In addition, an equivalent circuit of the HTS transformer is proposed to analyze the characteristic of the transformer. The calculated values based on the equivalent circuit are consistent with that of experiment. The detailed results about the design of the HTS partial-core transformer, the experiment, and the equivalent circuit are presented and discussed in this study.
\end{abstract}

\section{Introduction}

With the development of production technology of high temperature superconducting (HTS) tapes, HTS techniques have been applied to build electric power devices. As an important part of these devices, HTS transformer is expected to be one of the most promising application. It has several advantages, such as lower weight, smaller volume, higher efficiency and no fire risk. In [1], the HTS transformer fabricated by the authors has high efficiency of $99.4 \%$ at rating power 1 MVA and high performances for over-current of short circuit and lighting impulse voltage. However, the problem of high current, $\mathrm{AC}$ loss and the ability of superconductor limit its practical application and reduce its lifetime.

During the past decades, a number of HTS transformers have been designed [2]-[9]. There are three types of HTS transformers using different structures, fullcore, air-core and partial-core transformers are included. Most of the fabricated HTS transformers are full-core transformers, and it is a typical and practical structure. HTS air-core transformer has several disadvantages due to its air magnetic circuit, and a large amount of wires are needed to generate the proper magnetization current, which is not economical and has negative influence on its AC loss.
In the paper, we proposed a new type of HTS transformer, where the iron core is different in that the limbs and connecting yokes are absent. Details of the design of the HTS partial-core transformer including winding, core and Dewar are given. The results of the experiment and fundamental characteristics are also presented in the paper. In addition, an equivalent circuit of the HTS transformer is proposed to analyze the characteristic of the transformer.

\section{Transformer design}

The design of the HTS transformer includes its winding design, core design, Dewar design and assembly design. All the design involved computer modeling and empirical experimentation. Finite element simulation was used in the computer modeling.

\subsection{Winding design}

The HTS transformer was designed with two different kinds of windings using secondary generation (2G) HTS GdBCO wires. The primary windings are three layers connected in series, while the secondary windings are nine double pancakes connected in parallel, as shown in

$\bar{a}$ Corresponding author: seven840523@163.com 
Fig.1.

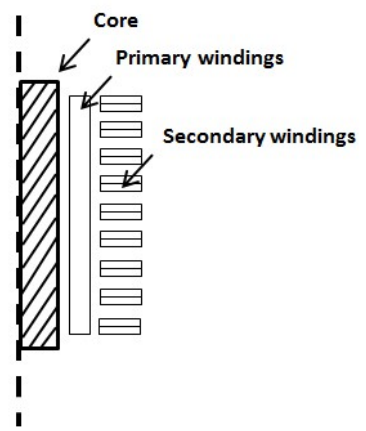

Figure 1. Cross-section view of partial-core transformer.

Fig. 2 shows the photographs of primary windings and the double pancakes. The critical current of the wire is $150 \mathrm{~A}$ at $77 \mathrm{~K}$ in a self field. However, the alternating magnetic fields present in the transformer resulted in a reduction in critical current. The critical current of primary windings became $100 \mathrm{~A}$ at $77 \mathrm{~K}$, while that of double pancakes coils reduced to $140 \mathrm{~A}$ at $77 \mathrm{k}$ on average.

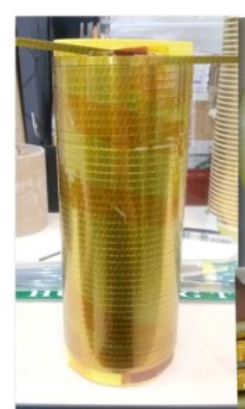

(a)

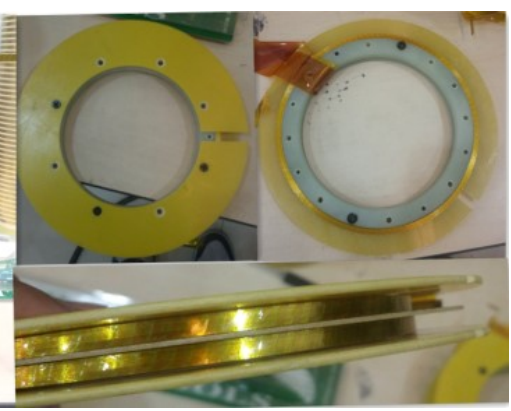

(b)
Figure 2. Photographs of the transformer windings. (a) Primary windings; (b) double pancakes.

The HTS wire was insulated turn to turn by using kapton. The thickness of kapton tape is $50 \mu \mathrm{m}$. The AC breakdown voltage of kapton was $118.6 \mathrm{kVmm}-1$ tested at a liquid nitrogen temperature of $77 \mathrm{~K}$, which is enough for windings in the transformer. Table 1 lists the specifications of the tapes and windings.

Table 1. Specifications of HTS tapes and windings.

\begin{tabular}{|c|c|c|}
\hline \multirow{4}{*}{$\begin{array}{l}\text { GdBCO } \\
\text { tape }\end{array}$} & Width & $4.2 \mathrm{~mm}$ \\
\hline & $\begin{array}{c}\text { Thickness (without } \\
\text { insulation) }\end{array}$ & $0.24 \mathrm{~mm}$ \\
\hline & Thickness (with insulation) & $0.34 \mathrm{~mm}$ \\
\hline & Critical current & $150 \mathrm{~A}$, at $77 \mathrm{~K}$ \\
\hline \multirow{5}{*}{$\begin{array}{l}\text { Primary } \\
\text { windings }\end{array}$} & Inner radius & $41 \mathrm{~mm}$ \\
\hline & Outer radius & $43 \mathrm{~mm}$ \\
\hline & Bobbin material & epoxy resin \\
\hline & Number of turns & 180 (45 / layer) \\
\hline & Insulation & Kapton tape \\
\hline \multirow{5}{*}{$\begin{array}{c}\text { Secondary } \\
\text { windings } \\
\text { (per } \\
\text { double } \\
\text { pancake) }\end{array}$} & Inner radius & $45 \mathrm{~mm}$ \\
\hline & Outer radius & $50 \mathrm{~mm}$ \\
\hline & Bobbin material & G10 \\
\hline & $\begin{array}{c}\text { Number of turns } \\
\text { Numbers of pancake }\end{array}$ & $\begin{array}{c}18(9 / \text { pancake }) \\
9\end{array}$ \\
\hline & Insulation & Kapton tape \\
\hline
\end{tabular}

\subsection{Core design}

The core is a cylindrical central core. Its length is 225 $\mathrm{mm}$ and its diameter is $60 \mathrm{~mm}$. It is made of several laminations of $0.3 \mathrm{~mm}$ silicon steel. The laminations were cut to different widths to provide a circular shape. Table II lists its specifications.

Table 2. Specifications of iron core

\begin{tabular}{|c|c|c|}
\hline \multirow{4}{*}{ Iron core } & Length & $225 \mathrm{~mm}$ \\
\cline { 2 - 3 } & Radius & $30 \mathrm{~mm}$ \\
\cline { 2 - 3 } & Material & Silicon steel \\
\cline { 2 - 3 } & Thickness of lamination & $0.3 \mathrm{~mm}$ \\
\hline
\end{tabular}

\subsection{Dewar design}

The Dewar consists of two parts, inner vessel and out vessel. The inner vessel is exposed to room temperature to put iron core and the outer vessel is an annular shape for containment of liquid nitrogen, as shown in Fig.3. The material of Dewar is G10, the same as that of the double pancake. It had been proved that G10 is a cold-tolerant material, so it is suitable to be used to manufacture LN2 container. To reduce the heat leak from side wall of Dewar, heating preservation cotton was used to cover the side wall. Table III lists the specifications of Dewar.

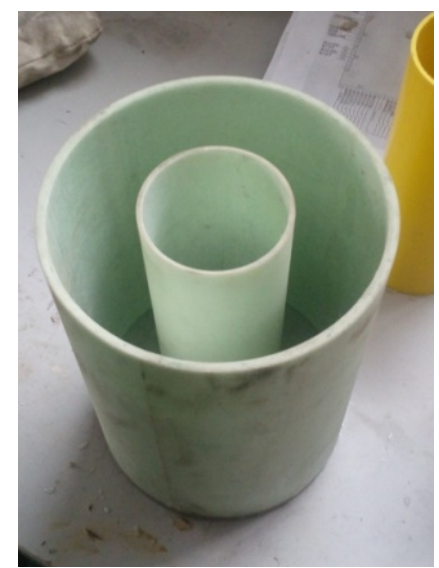

Figure 3. Photographs of the Dewar.

\section{Field-circuit coupled model}

To estimate the characteristics of the HTS transformer, a field-circuit coupled model was built using a finite element analysis software COMSOL Multiphysics. Apart from the geometry model of the transformer, Magnetic Field physics interface was used to simulate the magnetic field of the transformer, while an external circuit was connected to the windings using Electrical Circuit physics interface. A Time-Dependent study was used to calculate all relevant numerical values of transformer characteristics, including voltage, current, magnetic flux density and so on.

Fig.4 Field-circuit coupled model for HTS transformer simulation: (a) equivalent circuit model; (b) 3D model of HTS transformer built by COMSOL Multiphysics. 


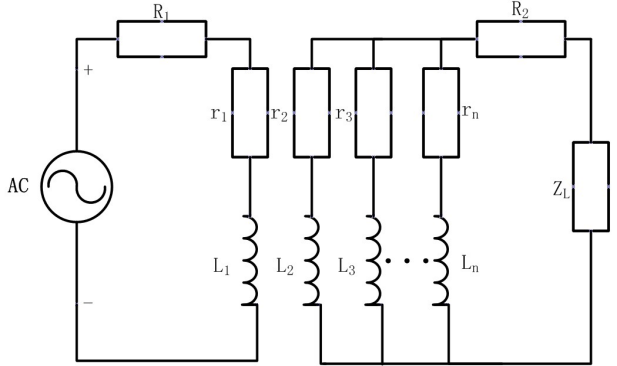

(a)

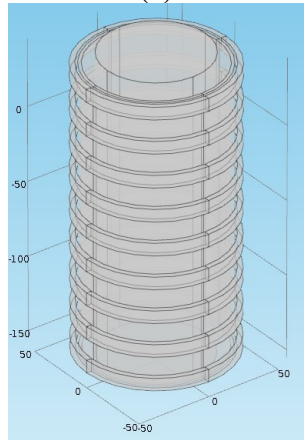

(b)

Figure 4. Field-circuit coupled model for HTS transformer simulation: (a) equivalent circuit model; (b) 3D model of HTS transformer built by COMSOL Multiphysics.

Fig.4 (a) shows the equivalent circuit model of the transformer and Fig.4 (b) shows the 3D model of HTS transformer built by COMSOL Multiphysics. In the 3D simulation model, the double pancakes are simplified to pancakes to benefit the calculation speed and each pancake are extremely uniform except the position. The governing equation of windings is expressed as (1), while that of iron core is expressed as (2).

$$
\begin{gathered}
\left\{\begin{array}{l}
\nabla \times\left(\mu_{0}^{-1} \mu_{r}^{-1} \vec{B}\right)-\sigma \frac{\partial \vec{A}}{\partial t} \times \vec{B}=\overrightarrow{J_{e}} \\
\vec{B}=\nabla \times \vec{A} \\
\overrightarrow{J_{e}}=\frac{N I_{\text {cir }}}{A} \overrightarrow{e_{\text {coil }}}
\end{array}\right. \\
\left\{\begin{array}{l}
\nabla \times \vec{H}-\sigma \frac{\partial \vec{A}}{\partial t} \times \vec{B}=\overrightarrow{J_{e}} \\
\vec{B}=\nabla \times \vec{A}
\end{array}\right.
\end{gathered}
$$

where $\mu_{0}$ is vacuum magnetic permeability, $\mu_{r}$ is relative permeability, $\sigma$ is electrical conductivity, $\boldsymbol{J}_{\boldsymbol{e}}$ is the surface current density, $N$ is the turn number, $\boldsymbol{I}_{\text {cir }}$ is the current of windings and $\boldsymbol{e}_{\text {coil }}$ is the direction of current.

According to the equivalent circuit model, the circuit equation for the primary and secondary windings can be expressed as (3).

$$
\left\{\begin{array}{l}
u_{1}=I_{1}\left(R_{1}+r_{1}\right)+e_{1} \\
u_{2}=e_{2}+i_{s} r_{s}-I_{2} R_{2} \\
u_{2}=I_{2} Z_{l} \\
I_{2}=-\sum_{s=2}^{n} i_{s}
\end{array}\right.
$$

where

$$
e_{k}=N_{k} \frac{d\left(\int \overrightarrow{A_{k}} \cdot d \overrightarrow{l_{k}}\right)}{d t}, k=1,2
$$

$\mathrm{R} 1$ is the primary resistance of the current lead, I1 is the primary current, $\mathrm{r} 1$ is resistance of primary windings, $\mathrm{e} 1$ is the primary induced voltage, is and rs are the branch current and resistances of secondary windings $(\mathrm{s}=2,3, \ldots, \mathrm{n}), \mathrm{R} 2$ is the secondary resistance of the current lead, I2 is the secondary current and $\mathrm{Zl}$ is the load. Because the windings are made of high temperature superconducting tapes, $\mathrm{r} 1$ and $\mathrm{rs}$ are equal to 0 .

The characteristics of the HTS transformer can be obtained by the simulation, such as open circuit test, load test and short circuit test, which would be compared to the experimental results in the following part.

\section{Experimental results}

All the experiments are made at temperature of liquid nitrogen at $77 \mathrm{~K}$, while the iron core is at room temperature.

\subsection{Open circuit test}

Open circuit test is carried out to determined the no-load exciting current and no-load loss of the HTS transformer. Experimental results of the no-load exciting current and no-load loss are shown in Fig.5, as well as the simulation result.
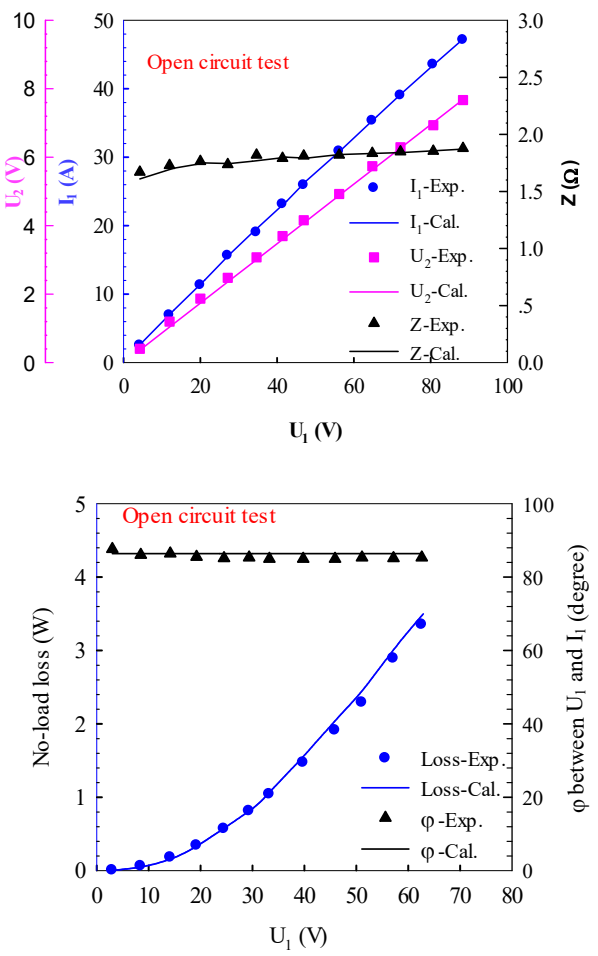

Figure 5. Experimental and calculated results of open circuit test. (a) I1, U2 and Z changing with U1; (b) No-load loss and difference between I1 and U2. 
As shown in Fig.5, I1 increases with U1 linearly, while the no-load loss rises proportionally with the square of $\mathrm{I} 1$. When $\mathrm{U} 1$ is $61 \mathrm{~V}$, the exciting current is $31 \mathrm{~A}$, noload loss is $3.3 \mathrm{~W}$. Apart from it, the experimental results and calculated results shows good consistency.

\subsection{Load test}

Load test is carried out to determined the HTS transformer's load characteristics and the distribution of current in the double pancakes. In the load test, $60-\mathrm{V}, 50$ $\mathrm{Hz} \mathrm{AC}$ voltage was applied to the primary windings.
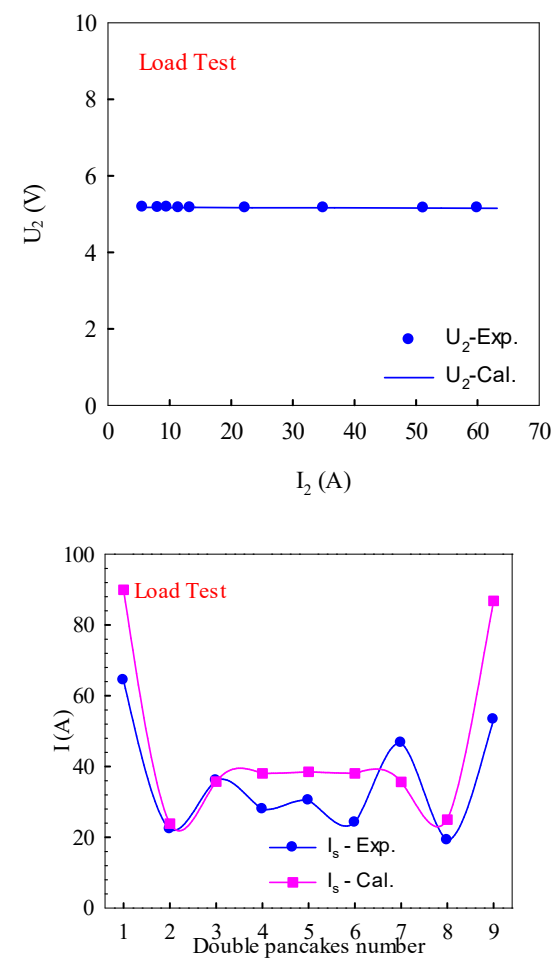

Figure 6. Experimental and calculated results of load test. (a) Load characteristics; (b) Distribution of current in double pancakes.

As shown in Fig.6 (a), with the increase of I2, U2 has a slightly decline. It indicates the load is resistive, which is correspond to the fact. The experimental and calculated results are also consistent. Fig.6 (b) shows the distribution of current in nine double pancakes. The number of the double pancakes was determined by their positions. No.1 represents its top-most position, while No.9 represents its lowest position. The experimental and calculated results show differences in the test. The calculated result shows high symmetry in the distribution of current in each double pancakes. Because the leakage reactance between branches are uneven, the distribution of branch current is inhomogeneous. No.1 and No.9 double pancakes have the highest current. From No.2 to No.5 double pancakes, the current in each double pancakes increases from $20 \mathrm{~A}$ to $40 \mathrm{~A}$, which is much smaller than that in No.1 and No.9 double pancakes, as well as that from No.8 to No.5 double pancakes. The experimental result shows a similar result as the calculated one, but there are some differences between the two results. The currents in No.1 and No.9 double pancakes are smaller than that of calculated result, though they are still larger than other double pancakes. Meanwhile, the symmetry of the current distribution is not obvious as the calculated result. As known, the tapes are resistant free at temperature of $77 \mathrm{~K}$, but the solder in weld part is not. The resistant of solder may have an important influence on the current distribution. Because the welding conditions are different, the distribution of current is affected.

\subsection{Short circuit test}

The short circuit test was operated with the HTS transformer's secondary windings shorted. A power transformer was adjusted to a target voltage with its switch open, whose secondary windings were connected to the HTS transformer's primary windings. When the switch was closed, a 5-cycle fault was simulated and the voltage and current of HTS transformer windings were measured. After the 5-cycle fault, the switch was opened automatically.

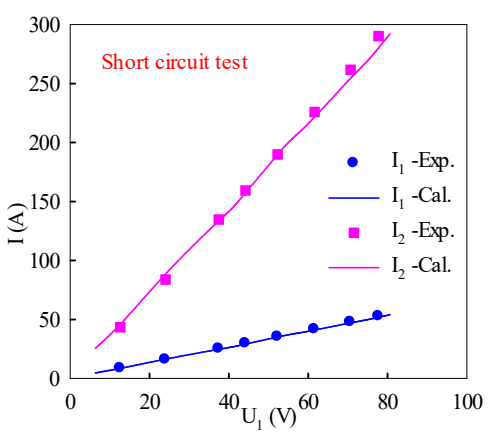

Figure 7. Experimental and calculated I1 and I 2 changing with U1

As shown in Fig.7, with the increase of U1, I1 and I2 increase linearly. It indicated that the HTS windings were at a superconducting state during the test time. When the primary voltage was $61 \mathrm{~V}$, the primary and secondary current were $41 \mathrm{~A}$ and $225 \mathrm{~A}$ respectively.

\section{Equivalent circuit}

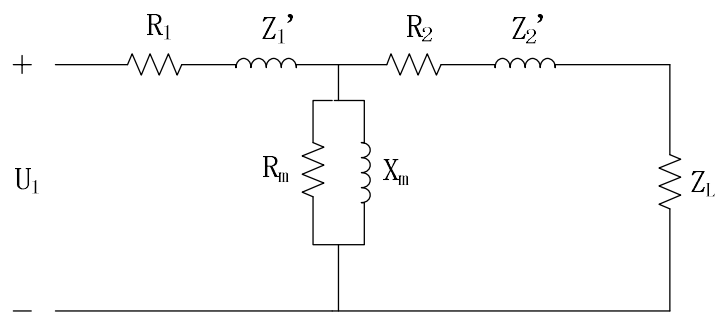

Figure 8. Equivalent circuit of HTS transformer, referred to the primary winding

Fig.8. shows the equivalent circuit of the HTS transformer referred to the primary winding. R1 and R2 are the resistances of current leads of primary and secondary winding respectively. $\mathrm{Z1}^{\prime}$ and $\mathrm{Z2}^{\prime}$ are 
impedances of primary and secondary windings. They are different from the common winding impedances as the equivalent resistances of loss are included. $\mathrm{Rm}$ and $\mathrm{Xm}$ are excitation resistance and reactance, respectively.

\section{Conclusion}

The paper presents the design and test of the HTS transformer with a cylindrical central iron core, made of GdBCO tapes. The cylindrical central iron core was a slug of laminated silicon steel. A Dewar was designed to contain the liquid nitrogen to provide the operation temperature for high temperature superconducting windings. Apart from these, the equivalent circuit with core loss was proposed, as well as the $3 \mathrm{D}$ field-circuit coupled model.

A serial electrical tests were performed on the transformer, such as open circuit test, short circuit test, load test, current distribution in double pancakes and critical current, and the results are consistent with the calculation ones. Although the no-load loss and voltage regulation percentage are qualified, as well as the ability of short current limiting, it has several problems indeed. Large exciting current and the irregular current distribution in secondary windings are the obvious problems. The vibration of the transformer at high voltage level is a tough problem, too. According to the result, the full core HTS transformer may be a better choice for transformer design, despite of a larger volume and heavier weight.

\section{Acknowledgments}

This paper is supported by Training and Financing Plan for Young University and College Teachers in Shanghai, Project No.: ZZGCD15109.

This paper is one of the periodical achievements made by the project Research on Semiology of China's Papercutting Animation (School Start-up 2016-24) carried out by Shanghai University of Engineering Science.
1. Funaki K, Iwakuma $\mathrm{M}$, Kajikawa $\mathrm{K}$, et al. Development of a $22 \mathrm{kV} / 6.9 \mathrm{kV}$ Single-Phase Model for a 3MVA HTS Power Transformer [J]. IEEE Trans.appl.supercond, 2001, 11 (1):1578-1581.

2. Baldwin, T.L, Ykema, J.I, Allen, C.L, et al. Design optimization of high-temperature superconducting power transformers [J]. IEEE Transactions on Applied Superconductivity, 2003, 13 (2): 2344-2347.

3. Tixador P, Donnier-Valentin G, Maher E. Design and construction of a $41 \mathrm{kVA} \mathrm{Bi} / \mathrm{Y}$ transformer [J]. IEEE Transactions on Applied Superconductivity, 2003, 13 (2): 2331-2336.

4. Glasson N, Staines M, Buckley R, et al. Development of a 1 MVA 3-Phase Superconducting Transformer Using YBCO Roebel Cable [J]. IEEE Transactions on Applied Superconductivity, 2011, 21 (3): 1393-1396.

5. Kim S H, Kim W S, Choi K D, et al. Characteristic tests of a 1 MVA single phase HTS transformer with concentrically arranged windings [J]. IEEE Transactions on Applied Superconductivity, 2005, 15 (2): 2214-2217.

6. Choi J, Lee S, Choi S, et al. Conceptual Design of a 5 MVA Single Phase High Temperature Superconducting Transformer [J]. IEEE Transactions on Applied Superconductivity, 2008, 18 (2): 636-639.

7. Lee S W, Byun S B, Kim W S, et al. Design of a Single Phase 33 MVA HTS Transformer with OLTC [J]. IEEE Transactions on Applied Superconductivity, 2007, 17 (2): 1939-1942.

8. Wang Y, Han J, Zhao X, et al. Development of a 45 kVA Single-Phase Model HTS Transformer [J]. IEEE Transactions on Applied Superconductivity, 2006, 16 (2): 1477-1480.

9. Qiu Q, Dai S, Wang Z, et al. Winding Design and Electromagnetic Analysis for a 1250-kVA HTS Transformer [J]. IEEE Transactions on Applied Superconductivity, 2015, 25 (1): 1-7.

10. Li X, Chen Q, Sun J, et al. Analysis of magnetic field and circulating current for HTS transformer windings [J]. IEEE Transactions on Applied Superconductivity, 2005, 15 (3): 3808-3813.

\section{References}

